\title{
Effects of Intensity and Position Modulation On Switched Electrode Electronics Beam Position Monitor Systems at Jefferson Lab*
}

\author{
Tom Powers \\ Thomas Jefferson National Accelerator Facility \\ Newport News, VA
}

\begin{abstract}
Two types of switched electrode electronics beam position monitors are in use at Jefferson Lab. Together they provide accurate beam position to the control system for beam intensities between $50 \mathrm{nA}$ and $2 \mathrm{~mA}$. One version, called the linac style, has a switching frequency of $125 \mathrm{kHz}$. The other, called the transport type, has a switching frequency of $7 \mathrm{kHz}$. The basic system provides information to the control system at a $1 \mathrm{~Hz}$ update rate. The systems are regularly used to measure the AC component of beam position and energy as well as suppress this motion as part of a fast feedback system. Position data produced by the system are also acquired on an event by event basis as part of the nuclear physics program in two of the experimental halls. This paper will focus on the AC characteristics of the system. These characteristics are affected by analog filter frequencies and a time delay between the measurement of the positive electrode signal and the negative electrode signal. The errors introduced by position and intensity modulation on the measured beam characteristics will also be discussed.
\end{abstract}

\section{INTRODUCTION}

The basic calculation for determining beam position for an opposing electrode based beam position monitor (BPM) system is the difference of opposing electrode voltages divided by the sum of the same signals times a constant. An imbalance in the gains between the two signals will introduce an error in the measurements. The switched electrode electronics (SEE) BPM system described in this paper overcomes this problem by employing a single amplifier-detector chain for each of the two $\mathrm{X}$ and $\mathrm{Y}$ channels. The opposing electrode signals are time domain multiplexed trough this amplifier chain. This was accomplished by switching the RF signals using a GaAs switching network as part of the front end electronics. The linac style, system design and the development of a second, more sensitive transport style system are described in [1] and [2].

The general specifications for the two types of systems are given in Table 1. The linac style SEE BPM systems are used primarily in the linacs as well as our free electron laser where they meet their specification for beam intensities between $0.5 \mu \mathrm{A}$

\footnotetext{
* Supported by DOE contract DE-AC05-84ER40150
} 
and $2000 \mu \mathrm{A}$. The transport type is used in the remainder of the CEBAF machine where it functions at beam intensities between $50 \mathrm{nA}$ and $200 \mu \mathrm{A}$. This BPM system provides information to the control system at $1 \mathrm{~Hz}$. A software tool called Bscope uses the data acquired by the BPM system to calculate the AC components of beam position and energy. Two groups of eight channels are used as part of a fast feedback system to suppress beam motion and energy jitter in the nuclear physics transport lines[3]. Position data generated by the system are also acquired on an event-by-event basis as part of the nuclear physics program in two of the experimental halls.

TABLE 1. System Specifications.

\begin{tabular}{lcc}
\hline Parameter & Linac Style & Transport Style \\
\hline Dynamic Range & $700 \mathrm{nA}-2 \mathrm{~mA}$ & $50 \mathrm{nA}-200 \mu \mathrm{A}$ \\
Nominal Update Rate & 1 measurement/second & 1 measurement/second \\
Position Range & $|\mathrm{x}|,|\mathrm{y}| \leq 5 \mathrm{~mm}$ & $|\mathrm{x}| \cdot|\mathrm{y}| \leq 5 \mathrm{~mm}$ \\
Resolution @ 1 Hz & $\leq 0.1 \mathrm{~mm}$ & $\leq 0.1 \mathrm{~mm}$ \\
Current Dependence & $\leq 0.1 \mathrm{~mm}$ & $\leq 0.1 \mathrm{~mm}$ \\
Multipass Capability & Yes & Not Required \\
IF Bandwidth & $1 \mathrm{MHz}$ & $50 \mathrm{kHz}$ \\
Gated Integrating Filter Bandwidth & $170 \mathrm{kHz}$ & $14.8 \mathrm{kHz}$ \\
Effective Analog Bandwidth & $57 \mathrm{kHz}$ & $3.6 \mathrm{kHz}$ \\
Due to Switching Effects & $114 \mathrm{kS} / \mathrm{s}$ & $7.1 \mathrm{kS} / \mathrm{s}$ \\
Maximum Data Rate (Positions) & & \\
\hline
\end{tabular}

\section{EFFECTS OF RASTERED BEAM}

In order to avoid target overheating, many of the experimental physics programs use a high frequency beam raster system in the last $30 \mathrm{~m}$ of beam line. Typical raster frequencies are between $15 \mathrm{kHz}$ and $25 \mathrm{kHz}$. Typical sinusoidal rastered beam motion is $4 \mathrm{~mm}$ peak-to-peak. Measuring average position, peak-to-peak beam motion, and instantaneous beam positions for this type of beam motion were not included in the original design specifications. As such, an analysis of the system was performed to better understand these effects.

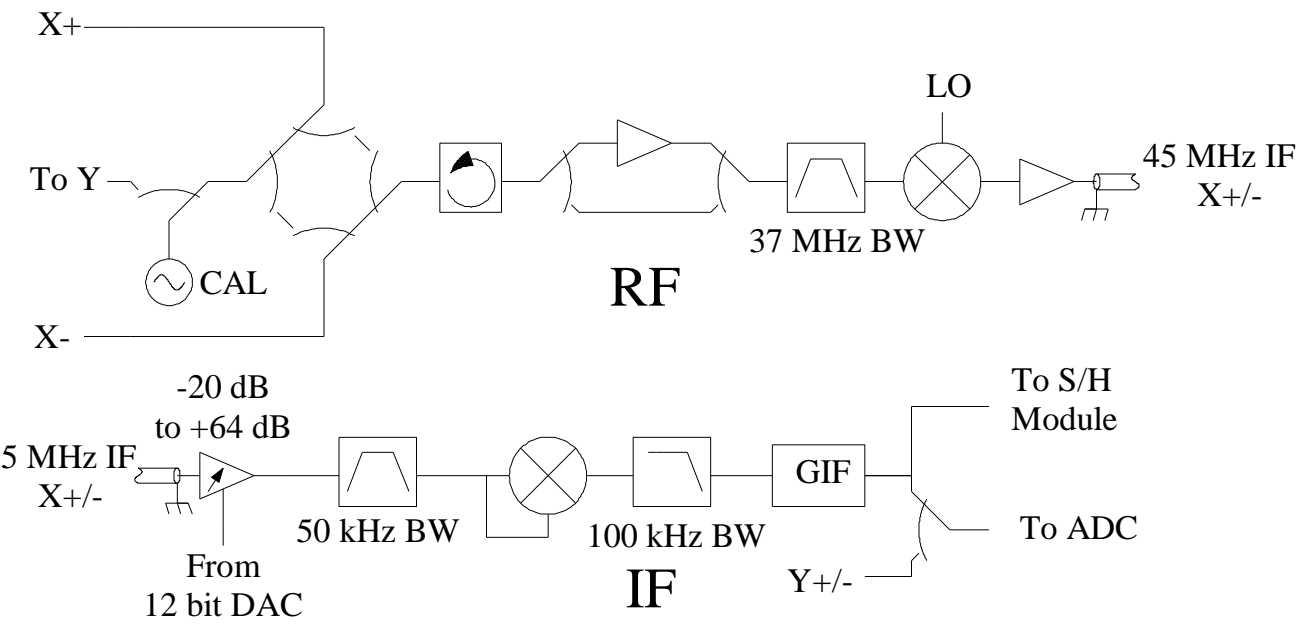

FIGURE 1. Block diagram of the transport style SEE electronics used at Jefferson Lab. 
A block diagram of the SEE electronics used in the CEBAF accelerator is shown in Figure 1. The dominant low pass filter used in the system is in the base band section. It is a gated integrating filter (GIF) with a $3 \mathrm{~dB}$ bandwidth of $170 \mathrm{kHz}$ for the linac style and $14.8 \mathrm{kHz}$ for the transport style. This type of filter provides good separation of the two electrode signals that are switched in the front-end circuitry. A gated integrating filter is characterized in the frequency domain by $\sin (\pi \mathrm{f} \Delta \mathrm{t}) / \pi \mathrm{f} \Delta \mathrm{t}$, where $\mathrm{f}$ is the frequency, and $\Delta \mathrm{t}$ is the integration time. Setting the value of $\sin (\mathrm{x}) / \mathrm{x}$ equal to 0.707 and solving yields a value of $\mathrm{x}=1.391$, or a $3 \mathrm{~dB}$ bandwidth of $0.443 / \Delta \mathrm{t}$. A switched BPM system is further complicated by the fact that alternate integration periods are combined to calculate each beam position. The timing of this switching, as well as that of the GIF filter, are shown in Figure 2. For the linac style system the integration time and sample intervals are $2.6 \mu$ s and $8.75 \mu$ s respectively, while for the transport style they are $30 \mu$ s and $140 \mu$ s respectively.
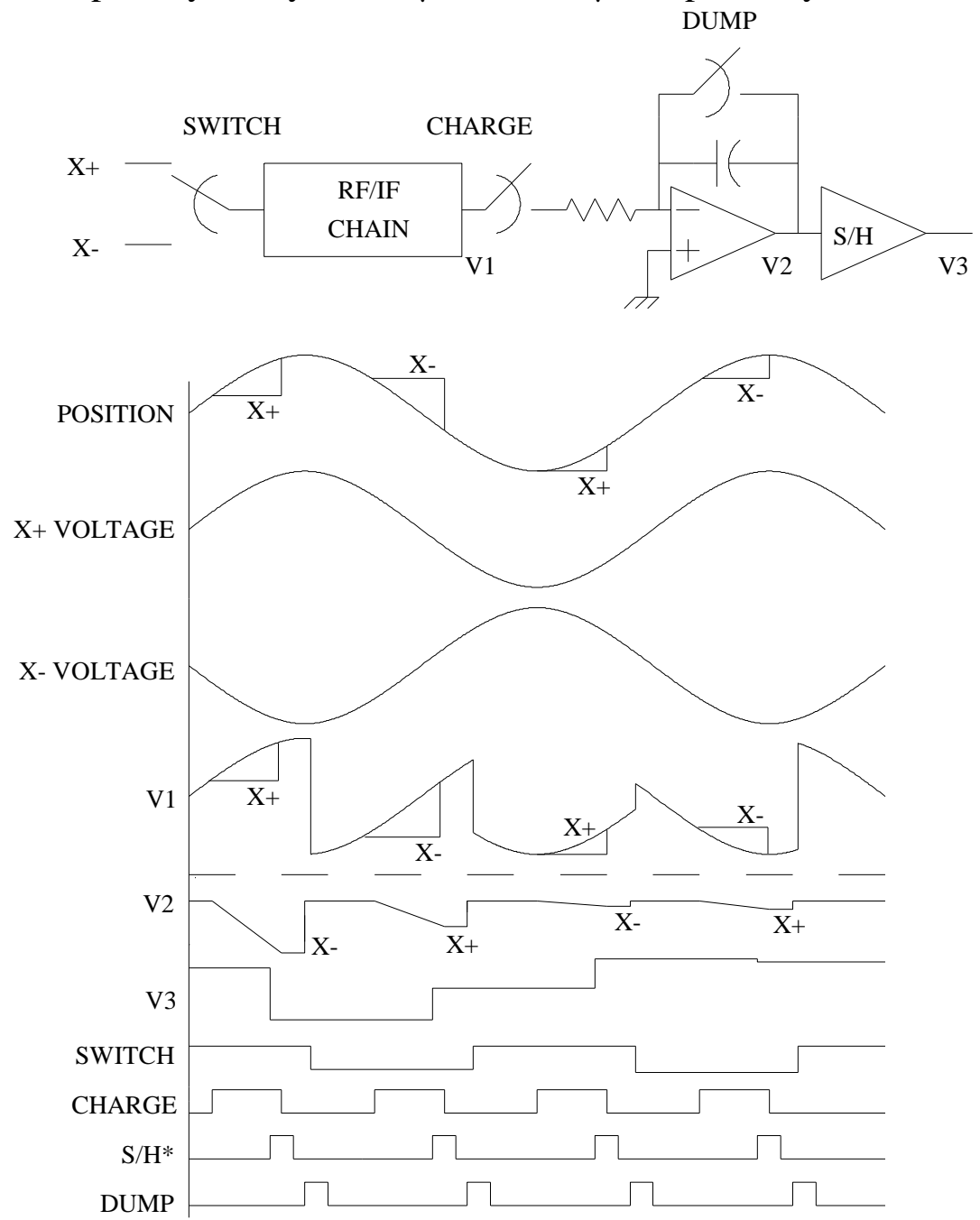

FIGURE 2. AC waveforms showing the integration and sampling times used for measuring beam position. For this example the frequency of the beam motion is approximately $40 \%$ less than the switching frequency. Note that V1 is always maintained between $1 \mathrm{~V}$ and $4 \mathrm{~V}$ by the automatic gain control circuit. 
A limited number of channels in the CEBAF machine are equipped with sample and hold modules, which de-multiplex and further filter the positive/negative electrode signals that are generated by the IF module. This is reasonable because the gain drifts, which led to the use of a switched system, are in the RF stages and the down conversion process. Once the signals are at baseband, gains and DC-offsets can be easily managed using a number of standard design practices. The final result of the de-multiplexing and filtering is the generation of four electrode signals for each BPM, which can be sampled simultaneously. These signals are typically sampled on an event-by-event basis during normal physics operations in Halls A and C.

A numerical simulation of the system was used to generate a transfer function for the particular timing of the linac and transport line BPM systems, as well as to investigate the effects of the $30 \mathrm{kHz}$ filter in the sample in hold module. The frequency response of the gated integrated filter in combination with the effects of the front end switching circuit are shown in Figure 3 which shows the relative gain of the system as a function of frequency. This is the frequency response that would be measured using the data acquisition system imbedded in the SEE electronics.

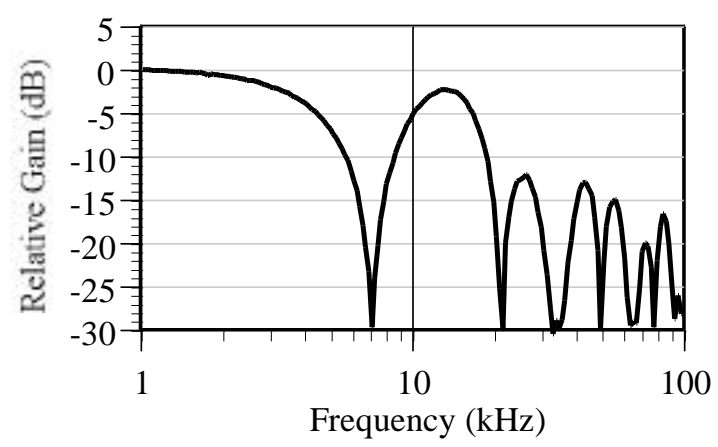

(a)

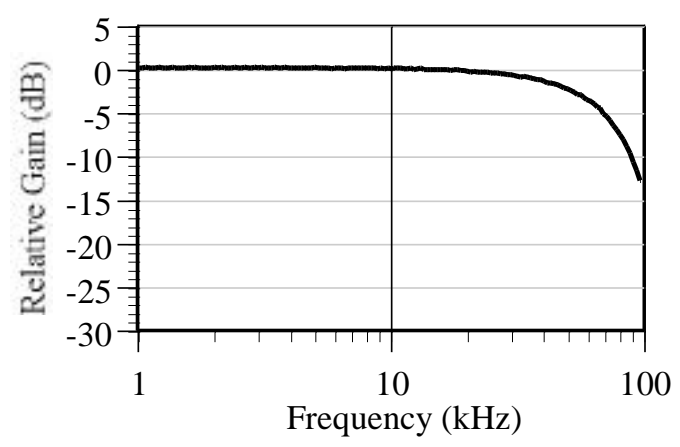

(b)

FIGURE 3. Frequency response of the gated integrating filter in combination with the effects of the front end switching electronics for (a) transport style SEE electronics and (b) linac style SEE electronics.

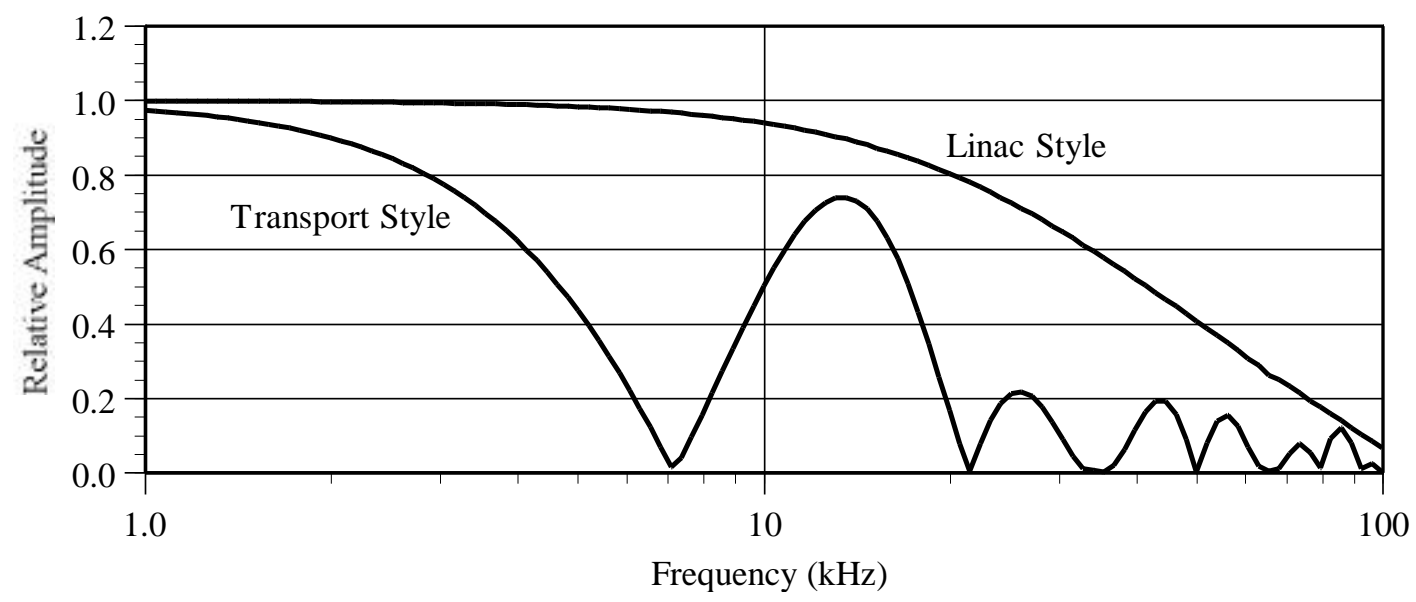

FIGURE 4. Predicted peak-to-peak amplitude for a rastered beam with a $1 \mathrm{~mm}$ pk-pk amplitude as measured using the outputs of the sample and hold module. The upper trace is the response of a linac style system and the lower trace is the response of a transport style system. 
Figure 4 shows the calculated values of the normalized peak-to-peak beam motion as a function of the frequency of the beam motion. The upper trace shows the results for the linac style system while the lower trace shows the results for a transport line system. These data include the effects of the $30 \mathrm{kHz}$ filter which is imbedded in the sample and hold module. The first zero observed in the transport line system response curves in Figure 3(a) and Figure 4 corresponds to the switching frequency of the SEE BPM electronics. At this frequency the two $30 \mu$ s sampling intervals occur at opposite polarities on the raster waveform, thus zero motion is measured. A similar zero response is observed in the linac style electronics at $124 \mathrm{kHz}$. Any measurements made for frequencies above the first zero will contain erroneous frequency content due to aliasing.

\section{INTENSITY MODULATION EFFECTS}

Position modulation in the $\mathrm{CW}$ beam is transformed into intensity modulation, measured using the sum of the electrode signals, due to the timing of the GIF and electrode switching. This coupling occurs because there is a time difference between measuring the positive and negative electrode signals, during which the position, and thus the electrode signal, changes. Similarly, intensity modulation is also transformed into a position modulation. Simulations of these effects were performed and the results are shown in Figures 5 and 6, respectively. Typically, the intensity modulation is less than $5 \%$ peak-to-peak, and the beam motion is seldom above $2 \mathrm{~mm}$ peak-to-peak in the CEBAF beam. The dominant frequency components are $60 \mathrm{~Hz}, 120 \mathrm{~Hz}$ and 180 $\mathrm{Hz}$, with the $180 \mathrm{~Hz}$ component contributing less than $20 \%$ to the motion and modulation. Under these conditions, the expected false beam motion would be less than $50 \mu \mathrm{m}$ and the false modulation contributions are less than $0.3 \%$ modulation. An analysis of the Bscope data of the BPMs in the CEBAF injector indicates that a 1.75 $\mathrm{mm}$ increase in beam motion yields an increase in the measured modulation by $0.23 \%$. No experiments have been performed to confirm the simulation results presented in Figure 5 and 6.

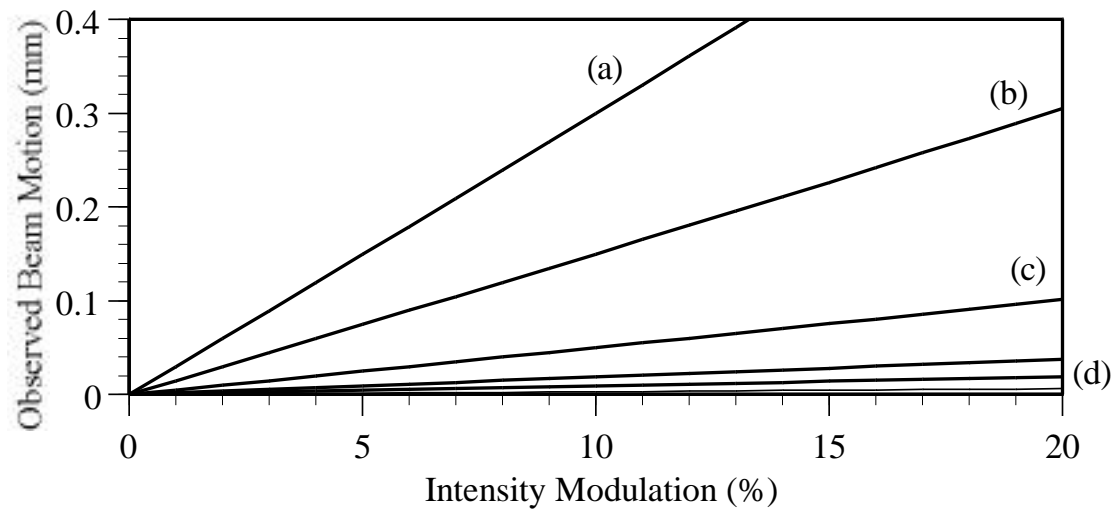

FIGURE 5. Observed beam motion as a function of intensity modulation. The data shown are for (a) transport style SEE BPM system with $360 \mathrm{~Hz}$ beam motion, (b) transport style with $180 \mathrm{~Hz}$ motion, (c) transport style with $60 \mathrm{~Hz}$ motion, (d) linac style with $360 \mathrm{~Hz}, 180 \mathrm{~Hz}$, and $60 \mathrm{~Hz}$ motion. 


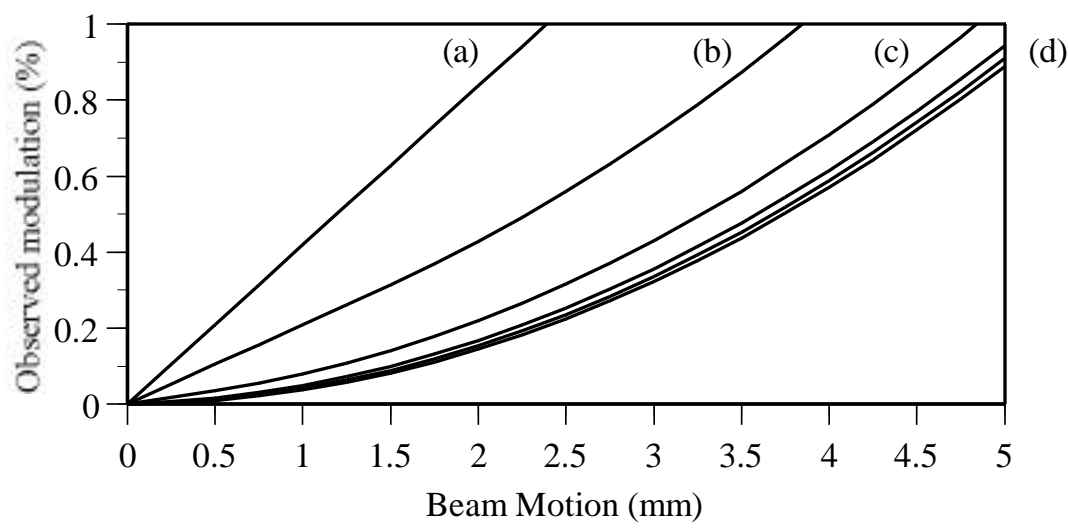

FIGURE 6. Observed intensity modulation as a function of beam motion for various frequencies of beam motion based on simulations. The data are for (a) transport style SEE BPM system with $360 \mathrm{~Hz}$ beam motion, (b) transport style with $180 \mathrm{~Hz}$ motion, (c) transport style with $60 \mathrm{~Hz}$ motion, (d) linac style with $360 \mathrm{~Hz}, 180 \mathrm{~Hz}$, and $60 \mathrm{~Hz}$ motion.

\section{CONCLUSIONS}

The major advantage of a switched electrode system is a reduction in the sensitivity to gain and offset drifts. This is accomplished through increased system complexity and a loss of high frequency capabilities. Experimental results show that the Jefferson Lab SEE-BPM systems meet their low current specifications of $50 \mathrm{nA}$ and $1 \mu \mathrm{A}$ operation for the transport style and linac styles, respectively. The results of simulations of the frequency response of the two types of systems were also presented. The linac style systems have a 3-dB bandwidth of $56 \mathrm{kHz}$. The addition of filtering, when they are equipped with the sample and hold modules, decreases this bandwidth to $27 \mathrm{kHz}$. The transport style systems have a bandwidth of $3.5 \mathrm{kHz}$. Because the systems acquire the different electrode signals at slightly different times, there is a coupling of beam position fluctuation and intensity. For a nominal $5 \%$ intensity modulation the false beam motion is expected to be less than $50 \mu \mathrm{m}$, while a $2 \mathrm{~mm}$ beam motion contributes less than a $0.3 \%$ intensity modulation.

\section{REFERENCES}

1. T. Powers, R. Ursic, L. Doolittle, and J. Wagner, "Design, Commissioning and Operational Results of Wide Dynamic Range BPM Switched Electrode Electronics", Proceedings of the Beam Instrumentation Workshop, Stanford, CA, (1998).

2. T. Powers, "Improvement of the Noise Figure of the CEBAF Switched Electrode Electronics BPM System", Proceedings of the Beam Instrumentation Workshop, Arogonne, IL. (1996)

3. V. Lebedev, and R. Dickson, "Fast Digital Feedback System for Energy and Beam Position Stabilization", Proceedings of the 1999 Particle Accelerator Conference, 